\title{
Numerical Computation of Flow in Porous Media of Non-Newtonian Power Law Fluids
}

\author{
Xiaoguang Liu ${ }^{1, a}{ }^{*}$, Xiaojun Zhou ${ }^{1, b}$, Xiaoru Hao ${ }^{1, c}$, Xiaohu Sang ${ }^{1, d}$ \\ ${ }^{1}$ School of Mechatronic Engineering and Automation, Shanghai University, Shanghai,China

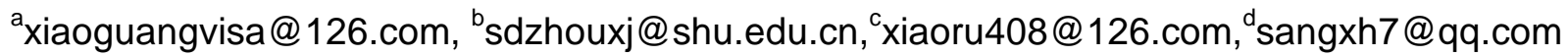

Keywords: power-law fluid, porous media, numerical computation

Abstract. In this article, the volume average method of fluid flow in porous media is introduced and the unit volume resistance is analysed, then the Brinkman-Forchheimer extended Darcy model is obtained. Numerical results of fluid flow in porous media are solved, numerical results show that fluid flow in porous media within the tube has obvious structural flow characteristics.Boundary layer viscous effect is limited to the vicinity of the wall surface, the results reflect the comprehensive effect of Darcy resistance, Forchheimer inertial resistance and Brinkman added resistance.Blocking effect of porous media has the velocity distribution of the tube flattened, dilatant fluid is more sensitive effect to this.

\section{Introduction}

Fluid flow in porous media is very complicated.Classic porous media fluid dynamics research Newtonian fluid ,but non-Newtonian fluids are widely used in engineering.

Non-Darcy flow in porous media include the low-speed non-Darcy flow and high-speed non-Darcy flow , most of the projects are the high-speed non-Darcy flow. Non-Darcy flow needs to be considered two main factors which are the inertial effect and non permeable boundary boundary layer effect.

Non-Darcy flow is also widespread in the fiber media and packed bed of the field of engineering, in this way, the flow of experimental observation often present a disordered state. To convert chaotic observations into definite quantitative relation, the appropriate mathematical physical model need to be established $^{[1-4]}$.

\section{Numerical Computation}

In this article, non Newtonian fluid flow problems in the porous media within the tube were solved numerically using the FLUENT software, we can obtain cross-section velocity distribution and pressure drop along the pipe axis value.

Firstly the problem of fluid flow in the uniform porous media is solved, We selection the fibrous material like foam metal,porosity is set to 0.98 , inertia coefficient CF is set to $0.11(0.05 \sim 0.17)$.

The velocity profiles of pseudoplastic fluid, Newton fluid and dilatant fluid in uniform porous media are shown in Fig.1, Fig.2 and Fig.3, respectively we can see the structural flow clearly from the figures for the whole block of solid matrix ${ }^{[6-8]}$. 


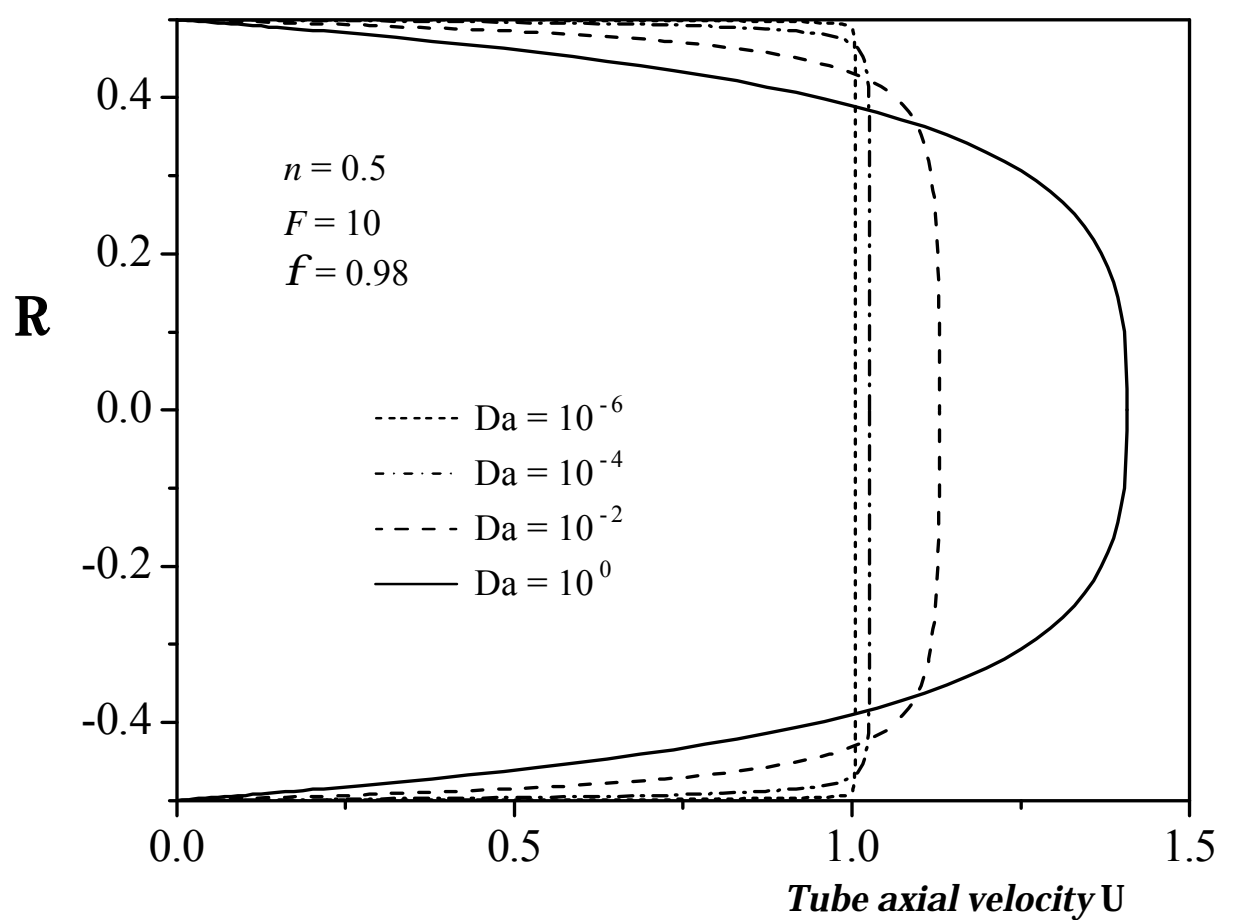

Fig.1 The axial velocity distribution of pseudoplastic fluid

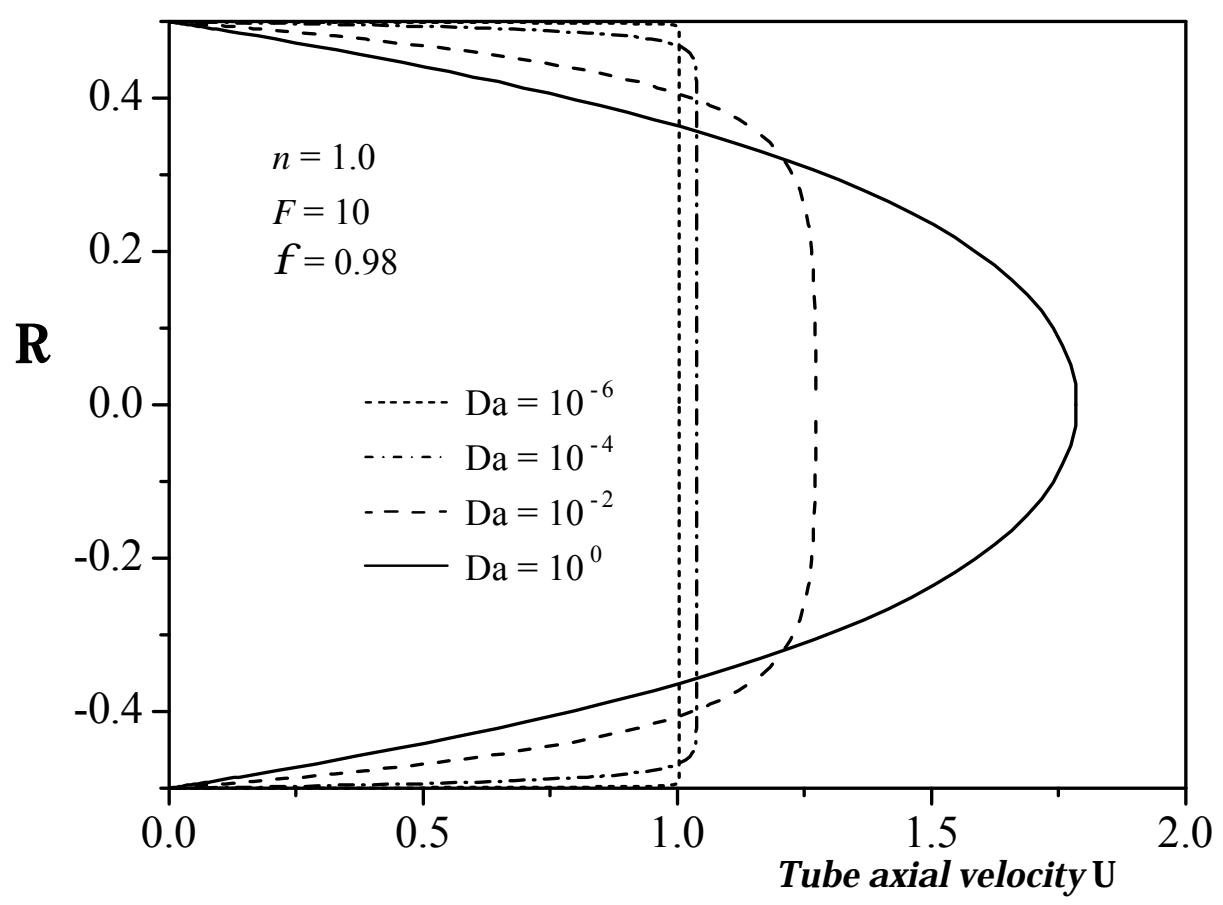

Fig.2 The axial velocity distribution of Newton fluid 


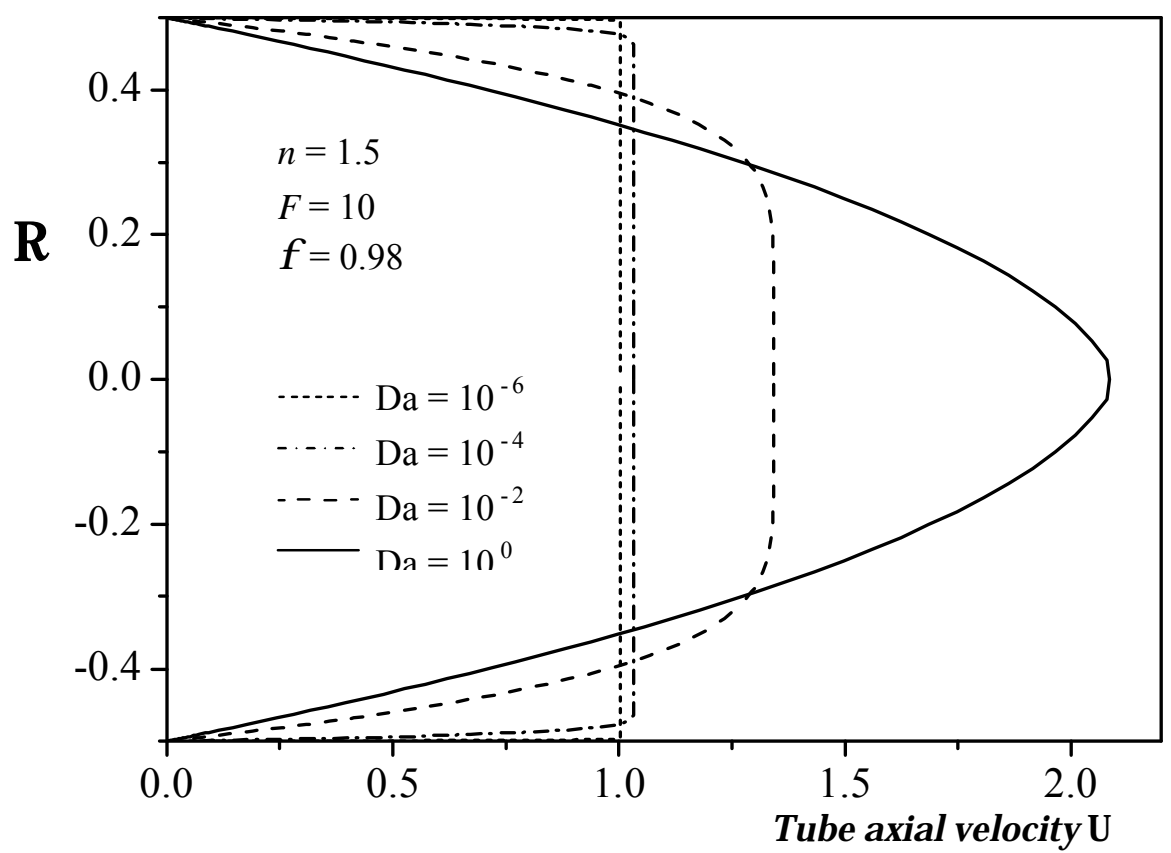

Fig.3The axial velocity distribution of dilatant fluid

The velocity profile of pseudoplastic fluid in general is more flatter than Newton fluid and dilatant fluid due to its characteristics of shear thinned, because the shearing acting of the pipe center and its surrounding fluid is weak, fluid near the pipe wall show up lower apparent viscosity by a large shearing acting, and thus the flow velocity near the pipe wall will be increased, so that make the boundary layer thin and have flow core zone increased.

The dilatant fluid near the wall become thickening by a strong shearing action, reduce the velocity of the boundary layer and increase the speed difference between the axis of pipe fluid and the wall fluid, the velocity profile shows significant parabolic characteristic.

With the decrease of the Darcy number, the whole block effect of porous media is more and more big, the velocity profile is also more and more flat, dilatant fluid is more sensitive effect to this.

The variation of the friction factor with Reynolds in packed bed is shown in Fig.4

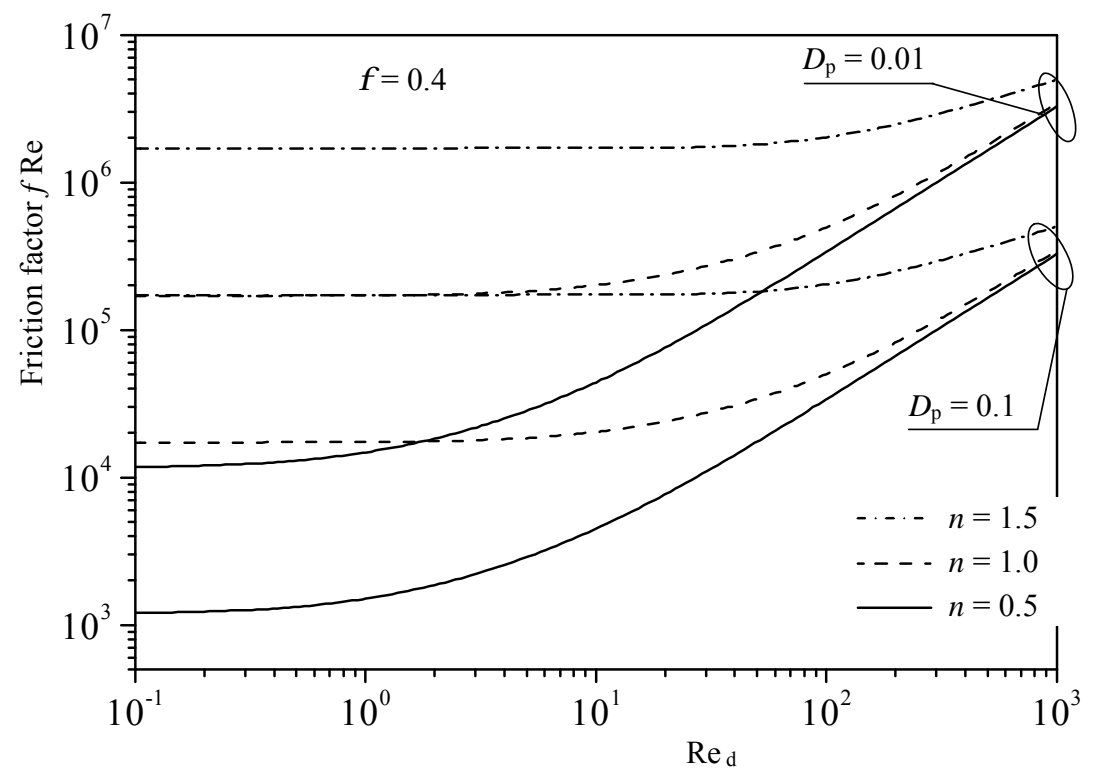

Fig.4 Variation of the friction factor with Reynolds in packed bed 
For the Darcy flow model (corresponding to the low Reynolds number), resistance factor $f \mathrm{Re}_{\mathrm{d}}$ is basically constant; As the increase of the Reynolds number, Darcy flow will change into Forchheimer flow and the resistance factor becomes bigger with the increase of the Reynolds number; Under the condition of high the Reynolds number, the inertia effect becomes very significant, and lead to resistance factor increases linearly with the Reynolds number.

\section{Conclusions}

(1)Introducing the volume average method of fluid flow in porous media and analysing unit volume resistance, then the Brinkman-Forchheimer extended Darcy model was obtained.

(2)Numerical solution of fluid flow in porous media is solved, numerical results show that fluid flow in porous media within the tube has obvious structural flow characteristics.

(3)Numerical results of fluid resistance show the method of volume averaging of flow in porous media and relevant empirical formula are effective. As in the aspect of applied basic theory research to lay a certain foundation.

\section{References}

[1] Liu S J, Masliyah J H. Non-linear flows in porous media. J Non-Newtonian Fluid Mech , 1999, 86: $229 \sim 252$

[2] Piau J M, Nigen S, Kissi N E. Effect of die entrance filtering on mitigation of upstream instability during extrusion of polymer melts. J Non-Newtonian Fluid Mech, 2000, 91: 37 57

[3] Spelt P.D.M,Yeow A.Y,Lawrence C.J,Selerland T.Creeping flows of Bingham fluids through arrays of aligned cylinders. Journal of Non Newtonian Fluid Mechanics, 2005

[4] James P W,Jones T E R,Hughes J P.The determination of apparent viscosity using a wide gap,double concentric cylinder. Non-Newton Fluid Mech, 2004

[5]VALDES-PARADAF.J.,OCHOA-TAPIAJ.A.andALVAREZ-RAMIREZJ.Validity of the permeability Carman-Kozeny equation: A volume averaging approach, Physica A:Statistical Mechanics and its Applications,2009,388(6):789-798.

[6] ROOS M.,BATAWI E. And HARNISCH U. et al. Efficient simulation of fuel cell stacks with the volume averaging method. Journal of Power sources, 2003,118(1-2):86-95.

[7] SIRIGNANO W.A.Volume averaging for the analysis of turbulent spray flows, International Journal of Multiphase Flow, 2005,31(6): 675-705.

[8] DAVIT Y., QUINTARD M. and DEBENEST G.Equivalence between volume averaging and moments matching techniques for mass transport models in porous media.International Journal of Heatand MassTransfer, 2010,53(21-22):4985-4993. 